MILITARY TECHNICAL COLLEGE CAIRO - EGYPT

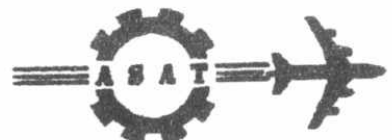

$7^{\text {th }}$ INTERNATIONAL CONF. ON AEROSPACE SCIENCES \& AVIATION TECHNOLOGY

\title{
REINFORCEMENT OF COMPOSITE ROCKET PROPELLANTS BY COTTON AND NITROCELLULOSE FIBRES
}

\author{
H.E.MOSTAFA ; S.M. HASSANIEN ${ }^{* *}$; M.A.RADWAN"* ; M..A.SADEK
}

\section{ABSTRACT}

The Egyptian extra long stapled cotton "GIZA76" which has a very good mechanical and chemical properties [1] was selected as a raw material to obtain nitrocellulose energetic fibres, such fibres can be used as a reinforcing agent in propellant formulations instead of inert fibres to improve not oniy the mechanical properties of composite propellants but also, the burning rate of such propellant. The effect of either chopped or nonchopped nitrocellulose fibres length and content on the mechanical properties of the prepared propellant samples were examined.Also, the effect of bonding agent on the reinforcing fibres has been investigated.Satisfactory results have been obtained.

\section{INTRODUCTION}

Improving the mechanical properties of composite rocket propellants is usually one of the main research goals, many programs have been carried out to avoid the effect of thermal and mechanical' stresses which are generated in the propellant grains due to long storing ,manipulation, case pressurization and firing. Many of these researches were focused on changing the type of ingredients and their weight percentage in the different rocket propellant formulations [2-4]. Many programs were concerned with the use of inert reinforcing fibres such as nylon and carbon fibres as reinforcing agents in many composite propellant formulations[5]. It is very important first to estimate the effect of reinforcement by inert cotton fibres then, reinforcement by energetic NC fibres on the processing, compatibility and on the mechanical properties of the polyurethane binder. A series of nonreinforced and reinforced propellant samples were prepared and tested. Nitrocellulose fibres either chopped $(10-15 \mathrm{~mm})$ or nonchopped $(30-50 \mathrm{~mm})$ [6] were to used to examine the effect of $\mathrm{NC}$ fibres length on the mechanical properties of reinforced propellants Also, the effect of the NC fibres content and that of the type and content of the bonding agent on the properties of the prepared samples have been investigated. All of the prepared samples were tested after complete curing by employing a modern tensile testing machine to obtain stress- strain relationship.

* Assistant Lecturer. Explosive Dpt. . MTC. Cairo. Egypt ** Assistant Professor. Head Of The Chem. Eng. Branch. MTC. Cairo. Egypt ** Assistant Professor. Hend Of The Explosive Dpt.. MTC. Cairo. Egypt *** Professor, Ex. Head Of Chem. Eng. Branch. MTC. Carru. Egypt 


\section{EXPERIMENTAL :}

\section{Materials}

All the chemical used were of high purity, their labeled specifications are listed in table (1)

Table 1. Raw Materials

\begin{tabular}{|c|c|c|}
\hline Name & Abbreviation & source \\
\hline Hydroxy terminated polybutadiene & HTPB & Iverise CO., Brazil \\
\hline Hexamethylene diisocyanate & HMDI & Aldrish co., England \\
\hline Dioctyl azelate & DOZ & AZC., Egypt \\
\hline Ferric acetyl acetonate & FeAAc & ROF co., England \\
\hline Nickel trioxide & NIO & Merc co., Germany \\
\hline Ammonium nitrate & AN & AZC., Egypt \\
\hline Methyl aziridine phosphinoxide & MAPO & AZC., Egypt \\
\hline
\end{tabular}

\section{PROCEDURE}

\section{A-Preparation Technique}

Plasticized polyurethane prepolymer was used as a binder in the prepared propellant formulations. A laboratory scale 0.5 liter thermostated glass mixer was used for such preparation. Then, mixer is connected to vacuum pump in order to obtain samples free from any air bubbles. At the beginning, the calculated weight of the prepolymers was fed together with one third of the $\mathrm{DOZ}$ plasticizer quantity into the mixing vessel. For about 20 minutes, mixing was kept continuously at $50{ }^{\circ} \mathrm{C}$ and $3 \mathrm{~cm} \mathrm{Hg}$ pressure to remove any air bubbles from the mixture. Then, the required amount of oxidizer with the remaining part of plasticizer was added to the reaction mixture and thoroughly stirred for 15 minutes under vacuum. Finally, at the normal temperature, the calculated amount of curing agent on the basis of $(\mathrm{NCO} / \mathrm{OH})$ ratio equal 1.2 and the FeAAc catalyst of $(0.1 \%)$ from the total charge weight were added to the reaction mixture and vacuum was continued for 5 minutes. Then the mixture was carefully casted in a dumbbell shape standard die, at the same time the reinforcing cotton or NC fibres were replaced and disturbed in the standard casting die in case of reinforcement using nonchopped cotton or NC fibres of (30-50 $\mathrm{mm}$ )length while in case of reinforcement using NC fibres of (10-15 mm) length ,chopped 
fibres were added during the course of the oxidizer addition .Curing of the casted formulations was carried out in an oven at $60^{\circ} \mathrm{C}$ for 7 days .

\section{B-Determination Of The Mechanical Properties Of The Prepared Composite Propellant Samples}

The value of stress-strain of the prepared samples were measured experimentally at normal temperature by employing the LRX-5K materials testing machine supplied by LLOYD INSRUMENT, UK. The tensile test was carried out for at least three samples for each prepared formulation and the mean value of the obtained results was recorded.

\section{RESULTS AND DISCUSSION}

1-Effect of reinforcement by cotton and NC fibers on the mechanical properties of polyurethane binder

For the preparation of polyurethane binder, HTPB prepolymer was used with HMDI curing agent on the basis of 1.2 curing ratio and FeAAc as a catalyst. Also, DOZ was used as a plesticizer, The fibre content in the prepared reinforced binder samples was $0.1 \%$ by weight .Composition of the prepared nonreinforced and reinforced polyurethane binder are given in table (2). The measured tensile properties for the prepared bindersamples are presented in Fig (1) and Fig (2).

Table 2 . Composition of nonreinforced and reinforced polyurethane $(\mathrm{PU})$ binder samples either by cotton or NC fibres

\begin{tabular}{|c|c|c|c|}
\hline Sample & PU1 & PU2 & PU3 \\
\hline HTPB & 75.76 & 75.76 & 75.76 \\
\hline HMDI & 8.46 & 8.46 & 8.46 \\
\hline FeAAC & 0.08 & 0.08 & 0.08 \\
\hline DOZ & 15.70 & 15.60 & 15.60 \\
\hline Cotton fibres & -- & 0.1 & - \\
\hline NC fibres & -- & - & 0.1 \\
\hline
\end{tabular}

PU1......... Nonreinforced polyurethane binder

PU2 ...... polyurethane binder reinforced by cotton fibres

PU3 ........polyurethane binder reinforced by nonchopped NC fibres 
As we notice, The reinforcement by such very small percent of the mentioned fibres appreciably increases the tensile strength and at the same time decreases the strain of the samples, that is due to, the stress applied to the binder matrix is transferred to the reinforcing fibres across the fibre-matrix interface and as a result of the difference in tensile strain between matrix and reinforcing fibres, shear stresses are induced around the fibres in the direction of fibre axis hence the fibre is stressed in tension so, cotton or NC fibres as a reinforcing agent contribute with the binder matrix to resist the applied load. Also, we notice that Cotton fibres improved the tensile properties slightly more than NC fibres, such result may be explained by the fact that during nitration of cotton, fibres become shorter due to the degradation action of the used concentrated mixed acids[" $]$.

\section{2-Effect Of Addition Of Bonding Agent (MAPO) On Tensile Properties Of The Reinforced Propellant Formulations}

The fibre-matrix interfacial bond is particularly important in the reinforced composite propellants. Such interfacial bond must be sufficient for load to be transferred from the matrix to the fibres[8]. (MAPO) was tried to achieve the required bonding effect between the used NC fibres and the composite propellant matrix, such bonding agents was added in small concentration, $(0.1 \%)$ with the samples containing $70 \% \mathrm{AN}$ oxidizer (containing $3.5 \% \mathrm{Ni}_{2} \mathrm{O}_{3}$ to prevent $\mathrm{AN}$ phase change during mixing [9]) and reinforced either by chopped or by nonchopped NC fibres. The prepared propellant samples composition is given in Table (3).

Table 3. Composition of the reinforced propellant samples using bonding agent

\begin{tabular}{|c|c|c|c|}
\hline Ingredients & M1 & M2 & M3 \\
\hline AN & 67.9 & 67.9 & 67.9 \\
\hline DOZ & 7.40 & 7.30 & 7.30 \\
\hline HTPB & 20.632 & 20.632 & 20.632 \\
\hline HMDI & 1.846 & 1.846 & 1.846 \\
\hline FeAAC & 0.022 & 0.022 & 0.022 \\
\hline NIO & 2.10 & 2.10 & 2.10 \\
\hline MAPO & 0.1 & 0.1 & 0.1 \\
\hline Chopped NC fibres & - & - & 0.1 \\
\hline Nonchopped NC fibres & - & 0.1 & - \\
\hline
\end{tabular}


M1..nonreinforced propellant sample with (MAPO) bonding agent.

M2..Propellant sample reinforced by nonchopped NC fibers with (MAPO) bonding agent M3..Propellant sample reinforced by chopped NC fibers with (MAPO) bonding agent.

Mechanical properties of the reinforced composite propellant either by chopped or nonchopped NC fibres on using $0.1 \%$ (MAPO) as a bonding agent are presented in Fig(3) and Fig (4). From these results one can notice a considerable increase in both the maximum stress and the strain of the reinforced samples when compared with the nonreinforced ones. Also, values obtained using the nonchopped NC fibres were higher than those associated with the use of the chopped NC fibres. It has been proposed that a bonding agent is used mainly to form a tough binder layer around both the oxidizer filler and the reinforcing fibers [10]. Bonding agents must fulfill the requirements of being adsorbed on the oxidizer surface and essentially insoluble in binder phase, therefore MAPO is a good bonding agent as it is able to make a good adhesion between both the oxidizer particles and the NC fibres with the binder matrix and to build a strong interfacial bond at the fibre-matrix interface. Since MAPO is free from the (-OH) groups, which may consume a part of (-NCO) groups, therefore the presence of (MAPO) builds a good interfacial bond and at the same time does not affect the crosslinking density. It is obvious that,(MAPO) is convenient bonding agent in presence of NC fibres in the reinforced composite propellant.

\section{3-Dependence Of Tensile Properties Of Reinforced Propellant Formulations On (MAPO) Bonding Agent Content}

To investigate the effect of the content of bonding agent on the mechanical properties of the prepared reinforced composite propellant samples, series of samples were prepared using different content of the bonding agent (MAPO) (from 0.1 up to $0.3 \%$ ). All the prepared samples contained $70 \% \mathrm{AN}$ oxidizer and reinforced by $0.1 \%$ chopped NC fibres. The prepared samples composition are shown in Table (4), the tensile properties of the tested samples are presented on Fig (5). Form these results, it is clear that the maximum stress of the reinforced propellant increases considerably about $(18 \%)$ and at the same time, the strain conversely decreases about $(13 \%)$ gradually by increasing the MAPO content. When the content of (MAPO) was more than $0.3 \%$, the viscosity markedly increased and the castability of the prepared samples become very poor. So, the addition of $0.1 \%$ up to $0.2 \%$ of (MAPO) improves the mechanical properties and kept the viscosity within the acceptable limits $(<100,000$ poise $)$ which permit a good potlife for the prepared propellant formulations. 
Table 4. Composition of the reinforced propellant samples with variable bonding agent content

\begin{tabular}{|c|c|c|c|c|c|}
\hline Ingredients Sample & MA1 & MA2 & MA3 & MA4 & MA5 \\
\hline AN & 67.90 & 67.90 & 67.90 & 67.90 & 67.90 \\
\hline DOZ & 7.30 & 7.25 & 7.20 & 7.15 & 7.10 \\
\hline HTPB & 20.632 & 20.632 & 20.632 & 20.632 & 20.632 \\
\hline HMDI & 1.846 & 1.846 & 1.846 & 1.846 & 1.846 \\
\hline FeAAC & 0.022 & 0.022 & 0.022 & 0.022 & 0.022 \\
\hline NIO & 2.10 & 2.10 & 2.10 & 2.10 & 2.10 \\
\hline MAPO & 0.10 & 0.15 & 0.20 & 0.25 & 0.30 \\
\hline Chopped NC fibres & 0.10 & 0.10 & 0.10 & 0.10 & 0.10 \\
\hline
\end{tabular}

\section{4-Effect Of The NC Fibres Content On Tensile Properties Of Composite Propellant}

To study the effect of the NC fibres content on the mechanical properties and processing technique of the prepared reinforced propellant samples, the content the chopped NC fibres was increased from 0.1 up to $0.5 \%(\mathrm{~N} 1-\mathrm{N} 4)$ in the previous formulations. Compositions of the prepared reinforced propellant samples are shown in Table (5). The tensile properties of the prepared samples shown in Fig (6). From these result, it is clear that, the tensile stress markedly increases up to a maximum at about $0.3 \% \mathrm{NC}$ and then it starts to decrease slightly with increase in the NC content this may be due to the possible existence of weak points in which the fibre-fibre contact prevails[11]. On the other hand, the strain values are increased slightly by increase of NC fibres content but still maintaining the acceptable strain values. That is may be due to increase of the number of not nitrated hydroxyl groups which may cause consumption of a part of isocyanate thus decreasing crosslinking density. For practical application, addition of 0.1 up to $0.2 \%$ chopped NC fibres to the composite propellant formulations is quiet sufficient for improving the tensile properties without facing the technological problems of mixing and casting. 
Table 5. Composition of the reinforced propellant samples containing variable chopped NC fibres content

\begin{tabular}{|c|c|c|c|c|}
\hline Ingredients Samples & N1 & N2 & N3 & N4 \\
\hline AN & 67.90 & 67.90 & 67.90 & 67.90 \\
\hline HTPB & 20.632 & 20.632 & 20.632 & 20.632 \\
\hline DOZ & 7.30 & 7.20 & 7.10 & 6.90 \\
\hline HMDI & 1.846 & 1.846 & 1.846 & 1.846 \\
\hline FeAAC & 0.022 & 0.022 & 0.022 & 0.022 \\
\hline NIO & 2.10 & 2.10 & 2.10 & 2.10 \\
\hline MAPO & 0.10 & 0.10 & 0.10 & 0.10 \\
\hline Chopped NC fibres & 0.10 & 0.20 & 0.30 & 0.50 \\
\hline
\end{tabular}

\section{CONCLUSION}

This work represents an approach for investigation of the role of energetic reinforcements in composite rocket propellants. On the basis of the results presented of the undertaken investigation, the following conclusions can be obtained:

1-Presence of either cotton or NC fibers causes an increase in the tensile stress of the prepared reinforced polyurethane binder.

2-Polyurethane binder has been preferred as a binding material to complete the investigation due to its convenient mechanical properties.

3-Cotton fibers provide higher tensile properties than those for NC fibers but NC fibers have been preferred since they are active and energetic.

4-Very small content of the (MAPO) considerably improve the tensile properties of the samples (about $18 \%$ increase in tensile stress and $13 \%$ increase in tensile strain).

$5-\mathrm{Up}$ to $0.3 \%$ of NC fibers is convenient otherwise another processing technique for inclusion of NC fibers must be employed. 


\section{REFERENCES}

[1] Cotton Research institute : Agriculture research center, Ministry of Agriculture, Giza, Egypt, (1992) .

[2] Anon; "Propulsion and propellants ",AMCP, 706-282, (1963)

[3] J. W. Lavitt ; USP. 3890175, (1975)

[4] R. Lautz ; USP. 3923564, (1975)

[5] L. M.. Katz; " Handbook Of Composites “, Ch. 9 , 481 (1989)

[6] R. Trotman ; "Dyeing and Chemical Technology of Textile fibers", sixth edition, New York,(1984).

[7] Urbanski ; "Chemistry and Technology of Explosives" V 3, Pergamon Press. Ltd., Headington Hill Hall , Oxford Ox Obw, England (1985)

[8] L. Dukrksen and J. Cohen. Brit. ; "Thermal Insulation " p- 326, $721,(1973)$.

[9] Kelly ; "Solid Propellant Mechanical Property Testing, Failure Criteria and Aging ." Chap. 8 in propellant Manufacture Hazards and testing, C. Boyars and K. Klager (Eds. ), Advances in Chemistry

[10] M.Tan, Y. D. Zhang; "Evaluation Of The Energetic Characteristics Of Polyurethane Propellant" Franhover, (1990)

[11] Klager , A. J. Dimilo ;"Rocket Propellants" in N. M. Bikales, "Encyclo. Polym. Sci. Tech." vol. 12, Inter science publishers div, John Wiley, Inc., N. Y., 105-39 (1970). 


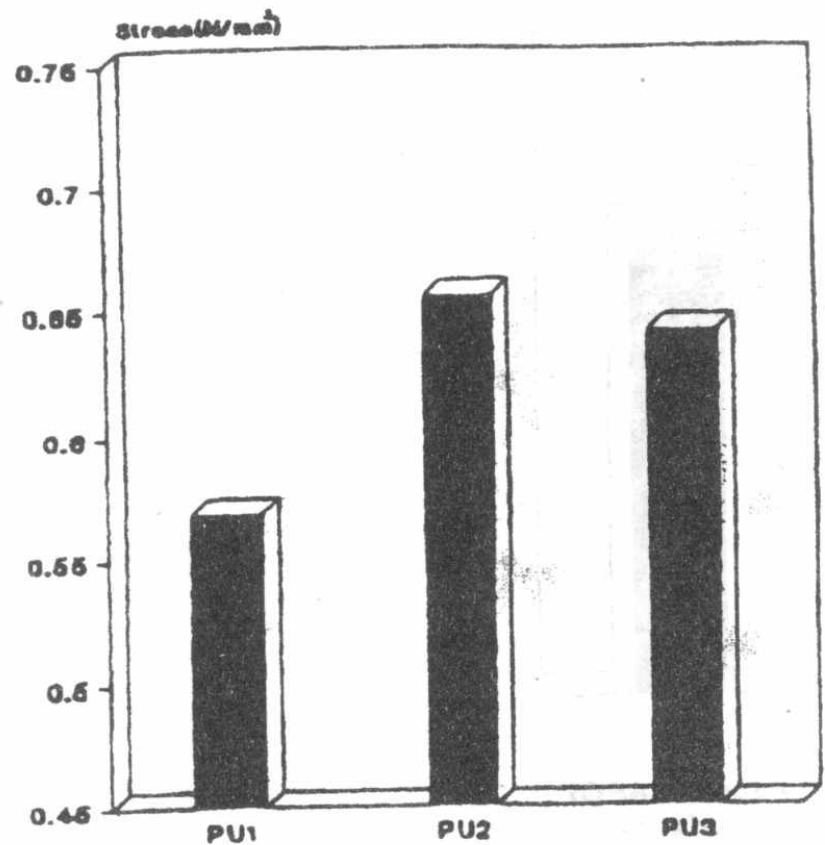

Fig ( 1 ) Tenalle Streas or Polyurethane Binder Peinforced By Cotron Or Nonchopped NC Flbres

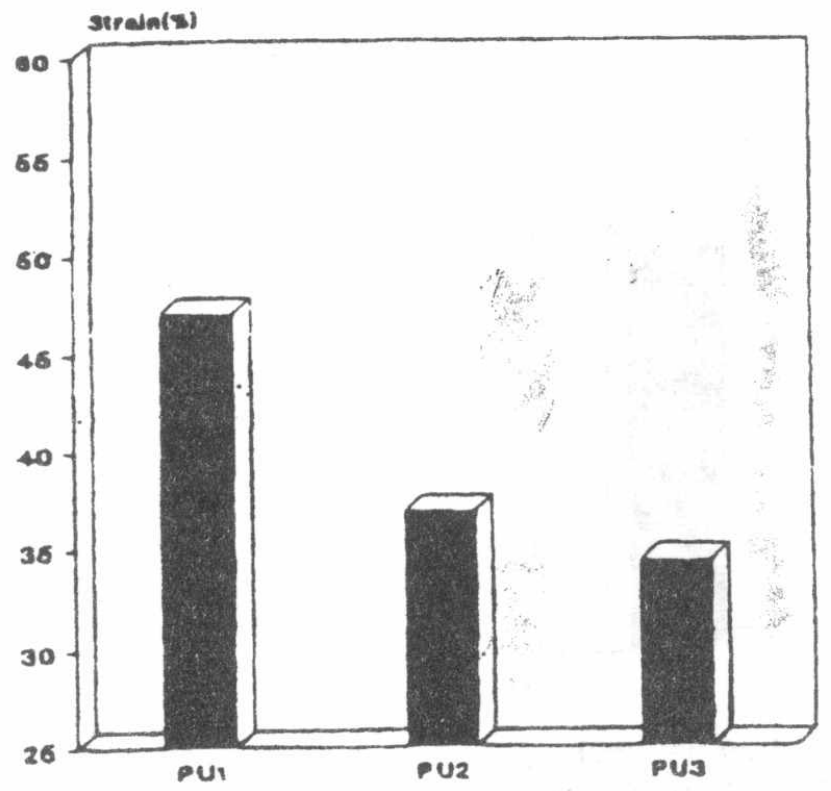

Fig ( 2 ) Tensile Strain Or Polyurethane Binder Relnforced By Cotton Or Nonchopped NC Fibres 


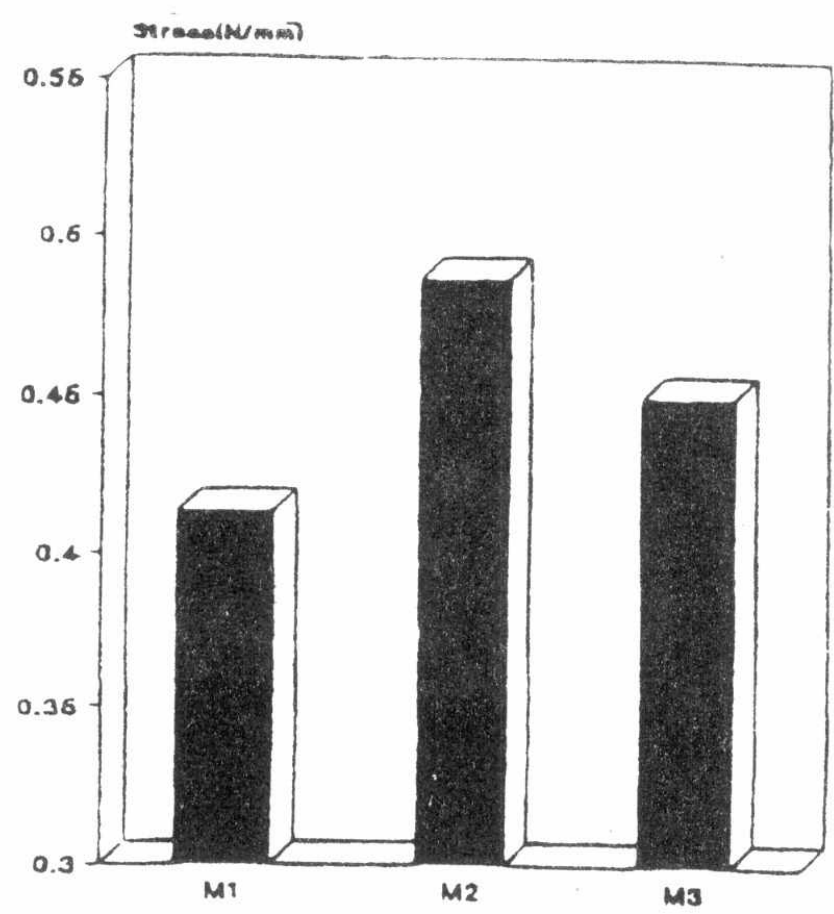

Fig ( 3 ) Effect Of Bonding Agent (MAPO) On Stress Or Reintorced Formulation

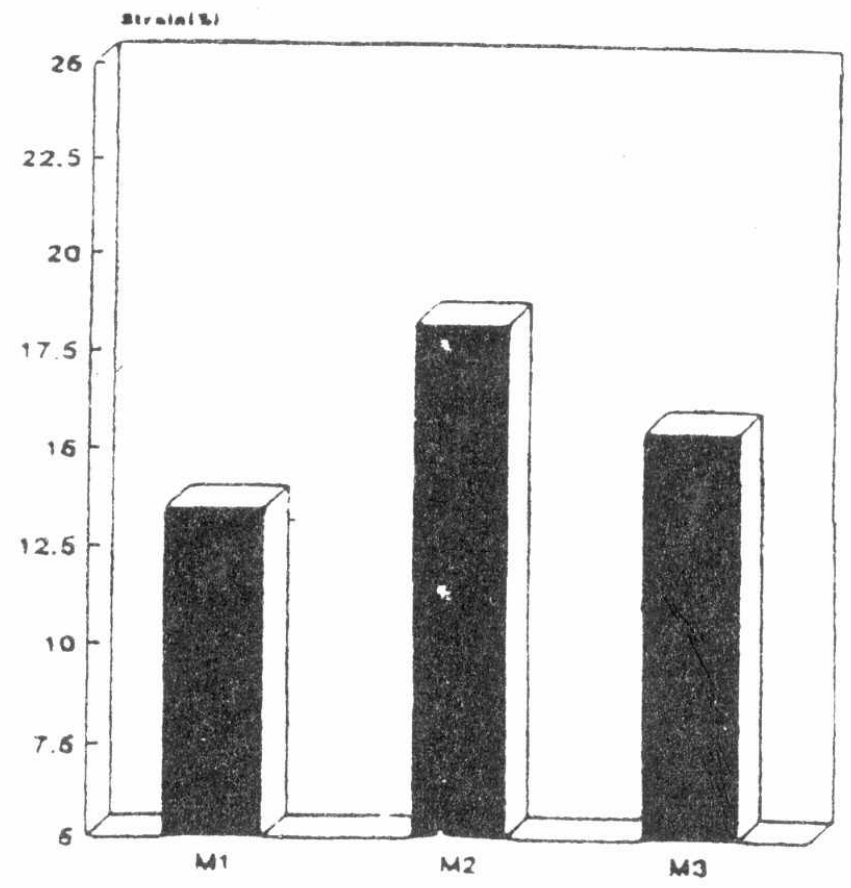

Fig ( +) Effoct of Bonding Agent(MAPO) On Strain Or Reinforced Formulation 


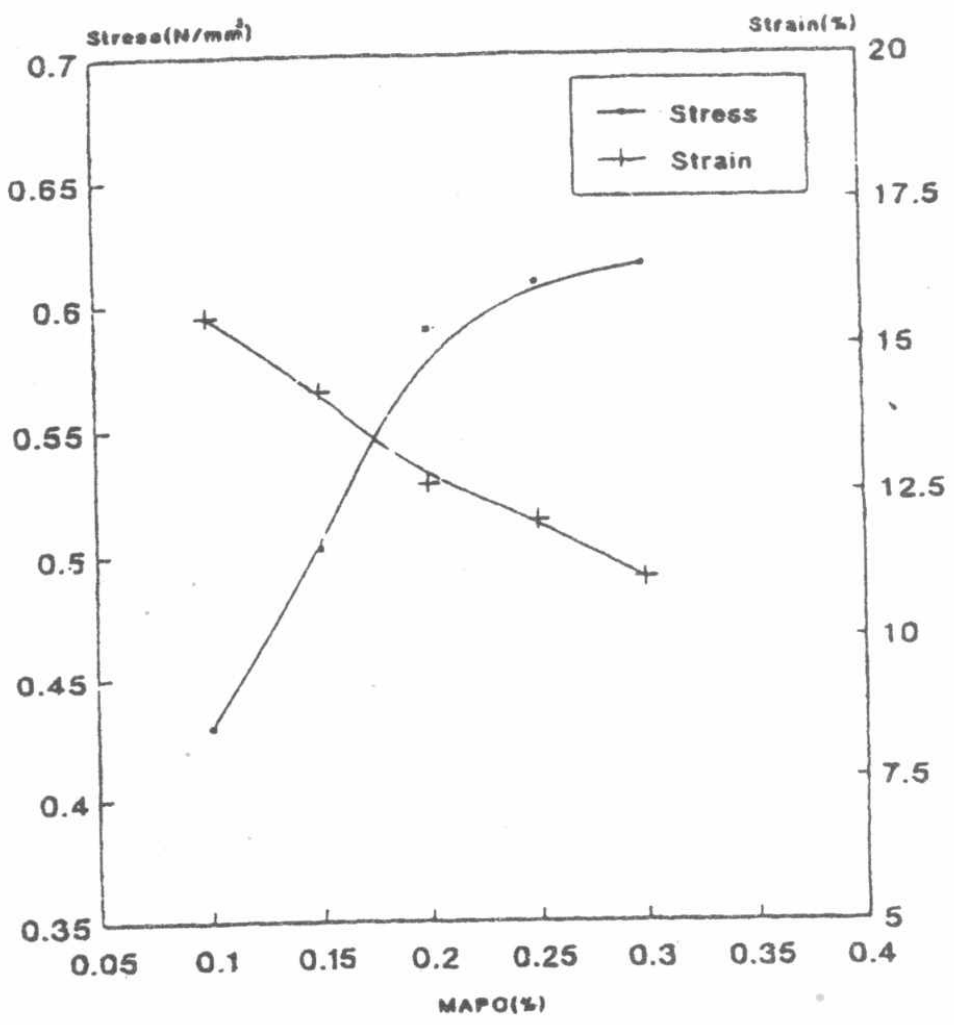

fig(5) Effect Of Bonding Agent (MAPO) Content On Tensile Properties Of feinforced Formulations

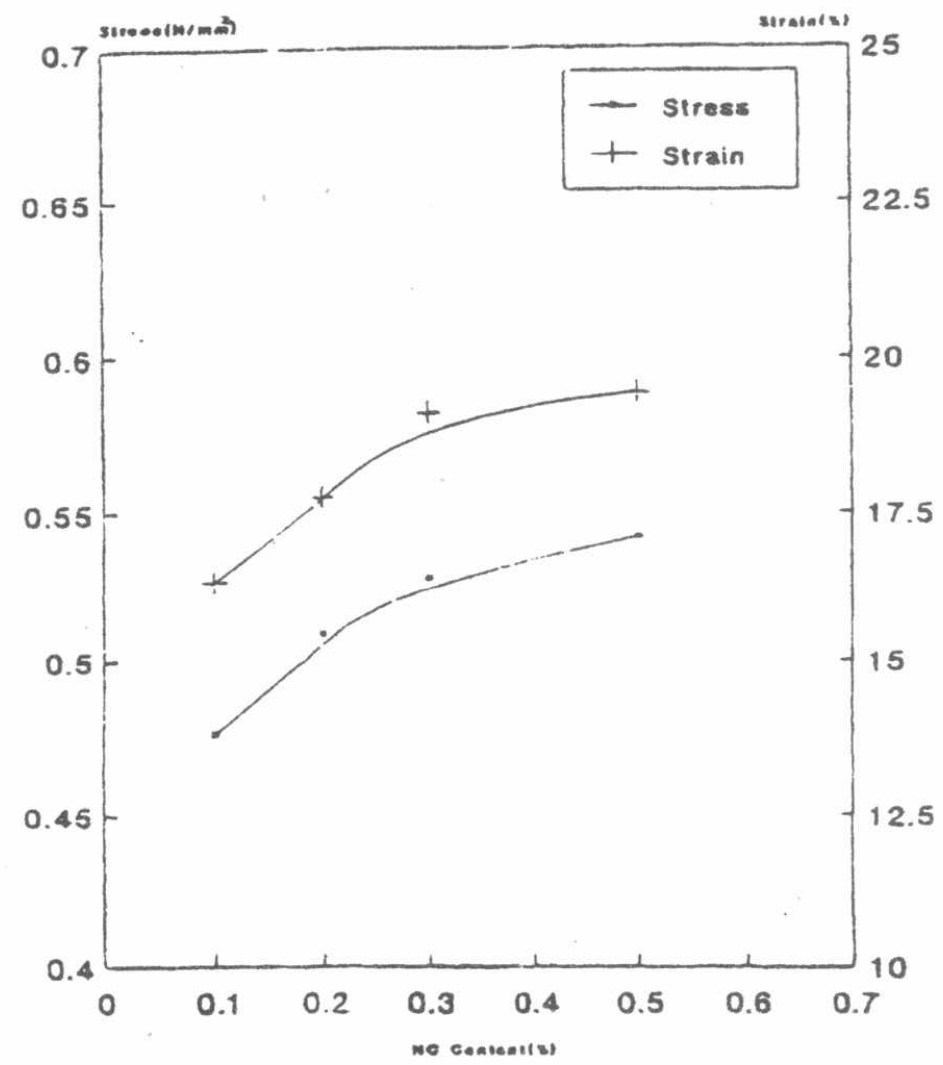

Fig(6) Effect Of Chopped NC Fibres On The Tensile Properties 